\title{
Papers
}

\section{Self reported cannabis use as a risk factor for schizophrenia in Swedish conscripts of 1969: historical cohort study}

\author{
Stanley Zammit, Peter Allebeck, Sven Andreasson, Ingvar Lundberg, Glyn Lewis
}

\begin{abstract}
Objectives An association between use of cannabis in adolescence and subsequent risk of schizophrenia was previously reported in a follow up of Swedish conscripts. Arguments were raised that this association may be due to use of drugs other than cannabis and that personality traits may have confounded results. We performed a further analysis of this cohort to address these uncertainties while extending the follow up period to identify additional cases.

Design Historical cohort study.

Setting 1969-70 survey of Swedish conscripts ( $>97 \%$ of the country's male population aged 18-20).

Participants 50087 subjects: data were available on self reported use of cannabis and other drugs, and on several social and psychological characteristics.

Main outcome measures Admissions to hospital for ICD-8/9 schizophrenia and other psychoses, as determined by record linkage.

Results Cannabis was associated with an increased risk of developing schizophrenia in a dose dependent fashion both for subjects who had ever used cannabis (adjusted odds ratio for linear trend of increasing frequency $1.2,95 \%$ confidence interval 1.1 to 1.4 , $\mathrm{P}<0.001$ ), and for subjects who had used only cannabis and no other drugs (adjusted odds ratio for linear trend $1.3,1.1$ to $1.5, \mathrm{P}<0.015)$. The adjusted odds ratio for using cannabis $>50$ times was 6.7 (2.1 to 21.7 ) in the cannabis only group. Similar results were obtained when analysis was restricted to subjects developing schizophrenia after five years after conscription, to exclude prodromal cases.

Conclusions Cannabis use is associated with an increased risk of developing schizophrenia, consistent with a causal relation. This association is not explained by use of other psychoactive drugs or personality traits relating to social integration.
\end{abstract}

\section{Introduction}

The relation between cannabis use and subsequent onset of psychosis is complex..$^{1-3}$ Although it is clear that high doses of cannabis may lead to a short lived toxic psychosis, it is unclear whether cannabis increases the risk of psychotic illness persisting after abstinence from the drug. An association between self reported use of cannabis in adolescence and subsequent risk of schizophrenia was reported from a cohort study of Swedish conscripts, ${ }^{4}$ which supports the view that cannabis might act as an independent risk factor for schizophrenia. Several uncertainties have, however, been raised regarding the interpretation of this result.

Firstly, the apparent effect of cannabis may be caused by other drugs (such as amphetamines) that are more likely to have been misused among cannabis users than among non-users. ${ }^{5}{ }^{6}$ Secondly, premorbid personality traits may have predisposed individuals both to developing schizophrenia and to using cannabis. Traits relating to social behaviour are likely to be particularly important in this respect. Thirdly, use of cannabis may have been secondary to the presence of schizophrenia, as a form of "self medication" for symptoms, despite failure to identify the disorder at the time of conscription. ${ }^{7}$ Review of case histories of a small subsample from this cohort shows that the association was not due to use of other drugs and that use of cannabis preceded any mental illness, ${ }^{8}$ but the causal pathways are difficult to disentangle and merit further study.

We are not aware of any other cohort studies that have investigated the association between drug use and subsequent risk of schizophrenia, and case-control studies are susceptible to recall bias. In this study we perform a further analysis of the Swedish conscript cohort to address some of the above concerns. ${ }^{4}$ The follow up period is now 27 years ( 15 years in the original study) and covers almost the whole period of risk for schizophrenia. ${ }^{9}$ Our improved understanding of risk factors for schizophrenia has also enabled us better to adjust for factors such as personality traits that potentially confound this relation..$^{10-13}$

\section{Methods}

Subjects

The cohort consisted of 50087 Swedish men conscripted for compulsory military training in 1969-1970. More than 98\% (49 321) were 18-20 years of age. Only $2-3 \%$ of the male population were excused conscription because of severe mental or physical handicap. The conscription procedure included intelligence tests and non-anonymous self reported ques- \begin{tabular}{l} 
Editorial by Rey and \\
Tennant \\
Papers pp 1195, \\
1212 \\
\hline \\
Department of \\
Psychological \\
Medicine, \\
University of Wales \\
College of \\
Medicine, Cardiff \\
CF14 4XN \\
Stanley Zammit \\
MRC clinical research \\
fellow \\
Department of \\
Social Medicine, \\
Gothenburg \\
University, Sweden \\
Peter Allebeck \\
professor of social \\
medicine \\
Department of \\
Public Health \\
Sciences, Karolinska \\
Institute, \\
Stockholm, Sweden \\
Sven Andreasson \\
associate professor of \\
social medicine \\
Ingvar Lundberg \\
professor of \\
occupational \\
epidemiology \\
Division of \\
Psychiatry, \\
University of \\
Bristol, Bristol \\
Glyn Lewis \\
professor of \\
psychiatric \\
epidemiology \\
Correspondence to: \\
S G Zammit \\
zammits@cardiff. \\
ac.uk \\
\end{tabular} 
tionnaires on family, social background, behaviour during adolescence, and substance use-including first drug used, drug most commonly used, frequency of use, and direct questions regarding use of a list of specified drugs. Details of the procedure and results of studies of its validity have been reported previously. ${ }^{14}$

All subjects underwent a structured interview conducted by a psychologist, and those reporting any psychiatric symptoms were interviewed by a psychiatrist and given a diagnosis according to ICD-8 (international classification of diseases, 8th revision) where applicable. ${ }^{15}$ Thirty four cases of psychosis diagnosed at conscription were excluded from the study. Permission to use the anonymised database was granted by the Karolinska Institute research ethics committee and the Swedish data inspection board.

\section{Follow up}

The Swedish national hospital discharge register recorded about $70 \%$ of all psychiatric admissions in 1970 , rising to $83 \%$ in 1973 . Coverage was $97 \%$ in $1974-83,95 \%$ in 1984-6, and has been virtually complete since 1987. The linkage reported here was from 1970 to 1996 . The incomplete registration during some periods is unlikely to have affected the results. Misclassification of outcomes is likely to be low, given that over $90 \%$ of people with schizophrenia are admitted to hospital at some point during their illness. ${ }^{16}$

Patients were given clinical diagnoses according to the Nordic version of ICD-8 (ICD-9 from 1987). Outcomes investigated were schizophrenia (codes 295.00-295.99) and other psychoses (including affective and paranoid psychoses, codes 296.00-298.99). It is unlikely that cases diagnosed as schizophrenia in this cohort were either toxic psychoses induced by cannabis (or amphetamine) or acute, transient drug induced psychoses, given the restrictive tradition in Sweden regarding the diagnosis of schizophrenia. ${ }^{17}$ Satisfactory validity of schizophrenia diagnoses in a small sample from this cohort has been observed, ${ }^{8}$ and ICD-8 diagnoses from the register have shown high specificity with criteria for schizophrenia as defined in DSM-III (Diagnostic and Statistical Manual of Mental Disorders, third edition). ${ }^{18}$

\section{Analysis}

We used logistic regression to calculate odds ratios and $95 \%$ confidence intervals for developing schizophrenia in subjects who used cannabis compared with subjects with no history of drug use, both before and after adjustment for potential confounders. Odds ratios may be interpreted as rate ratios because schizophrenia is a rare outcome. ${ }^{19}$ Although a few subjects died during follow up, analysis by using Cox regression made no difference to the results obtained by using logistic regression, which we therefore retained as the method of choice.

Previous research has found that psychiatric diagnosis at conscription, IQ score, personality variables concerned with interpersonal relationships, place of upbringing, paternal age, and cigarette smoking are all associated with schizophrenia. ${ }^{10-1320} 21$ We included these variables as potential confounders in the regression model. Disturbed behaviour in childhood, history of alcohol misuse, family history of psychiatric illness, financial situation of the family, and father's occupation were also considered to be potential confounders and included in the analysis. The variable relating to poor social integration as an aspect of personality was a summed score of questions regarding number of close friends, history of relationships with girlfriends, and individual sensitivity. We selected these questions after a factor analysis of over 40 questions relating to childhood and adolescent behaviour from one of the questionnaires. Only $3 \%$ of the sample had missing data for any of the questions.

Subjects were stratified into those receiving a diagnosis within five years of conscription ( $0-5$ years) and those receiving a diagnosis after this time ( $>5$ years) to investigate possible effects of a prodrome at the time of conscription.

\section{Results}

Out of the 50053 subjects, $362 \quad(0.71 \%$, 95\% confidence interval $0.65 \%$ to $0.80 \%$ ) received a diagnosis of schizophrenia by 1996. Data on drug use, derived from all sources of information, were missing on 16 $(4.4 \%)$ of subjects developing schizophrenia and on $1522(3.1 \%)$ of non-cases $(\mathrm{P}<0.2)$.

Of 11 variables initially included as potential confounders, only five had any effect on the adjusted results. Table 1 shows a summary of these in relation to cannabis use. Adjusting for poor social integration made minimal difference to results but is also included in table 1 . For the purposes of table 1 only, we treated IQ score, poor social integration, and disturbed behaviour as dichotomous variables, using the 10th percentile as a cut-off point for coding.

\section{Ever used cannabis}

Altogether 5391 subjects (10.8\% of the cohort) had ever used cannabis, and 73 of these (1.4\%) developed schizophrenia. In 69 subjects who started using drugs before 1969, 19 (31\%; 95\% confidence interval 20\% to $44 \%$ ) of those developing schizophrenia had stopped using drugs before conscription, as opposed to 2810 $(64 \% ; 62 \%$ to $65 \%)$ of the 4418 who did not develop schizophrenia $(\mathrm{P}<0.001)$.

Table 1 Summary of confounders in relation to subjects' history of drug use at conscription. Values are numbers (percentages) of cohort sample

\begin{tabular}{|c|c|c|c|c|c|c|}
\hline Subjects by type of drug use & $\begin{array}{l}\text { Diagnosis of } \\
\text { psychiatric illness on } \\
\text { conscription* }\end{array}$ & $\begin{array}{l}\text { Disturbed } \\
\text { behaviour }\end{array}$ & Low IQ score & Brought up in city & Cigarette smoking & $\begin{array}{l}\text { Poor social } \\
\text { integration }\end{array}$ \\
\hline Cannabis $(\mathrm{n}=5391)$ & $1408(27)$ & $1582(31)$ & $297(6)$ & $2262(43)$ & $4582(86)$ & $130(2.5)$ \\
\hline Cannabis only $(\mathrm{n}=1648)$ & $235(15)$ & $271(17)$ & $65(4)$ & $653(40)$ & $1331(81)$ & $34(2.1)$ \\
\hline Any drug $(\mathrm{n}=11783)$ & $2325(20)$ & $2557(23)$ & $1007(9)$ & $3358(31)$ & $8835(80)$ & $246(2.3)$ \\
\hline No drug $(n=36753)$ & $2827(8)$ & $1681(5)$ & $3510(10)$ & $6759(19)$ & $19229(52)$ & $841(2.4)$ \\
\hline
\end{tabular}

Owing to missing data for each of the confounders, the precentages presented may not tally precisely with the numbers of subjects reported

*Except psychosis or learning disability. 
The crude and adjusted odds ratios with 95\% confidence intervals for developing schizophrenia given a history of ever using cannabis are presented in tables 2 , 3 , and 4. The crude odds ratio for developing schizophrenia any time after conscription was 2.2 (1.7 to 2.8) and this association persisted, although reduced, after adjustment (adjusted odds ratio 1.5, 1.1 to 2.0).

We found a dose dependent relation between frequency of cannabis use and risk of schizophrenia, with an adjusted odds ratio for linear trend across the categories of frequency of cannabis use used in this study of 1.2 (1.1 to $1.4, \mathrm{P}<0.001)$. The adjusted odds ratio for subjects with a history of heaviest use of cannabis ( $>50$ occasions) was 3.1 (1.7 to 5.5 ).

The association between cannabis use and schizophrenia was greater in subjects admitted in the first five years after conscription (adjusted odds ratio 2.1, 1.2 to 3.7) compared with those admitted after five years (1.2, 0.8 to 1.8). Frequency of cannabis use was associated with schizophrenia in both the early onset group (adjusted odds ratio for linear trend 1.3, 1.1 to 1.6, $\mathrm{P}<0.001)$ and the later onset group (1.2, 1.1 to 1.3, $\mathrm{P}<0.02)$.

\section{Cannabis only}

Altogether 1648 subjects $(3.3 \%$ of cohort, $3.1 \%$ to $3.5 \%)$ had used only cannabis, and 18 of these $(1.1 \%$, 0.6 to $1.7 \%$ ) developed schizophrenia. Those who used only cannabis had an increased risk of schizophrenia compared with those who reported no drug use. The odds ratio before adjustment $(1.9,1.2$ to 3.0$)$ and afterwards $(1.9,1.1$ to 3.1) was similar (table 5). We found a dose dependent relation for frequency of use, with an adjusted odds ratio for linear trend of 1.3 (1.0 to 1.5, $\mathrm{P}<0.02)$.

\section{Stimulant use}

We found an association between schizophrenia and stimulant use in the crude analysis (crude odds ratio 3.8, 2.7 to 5.4), but this became non-significant after adjustment for confounders (adjusted odds ratio 1.5, 0.9 to 2.4). Adjusting for frequency of cannabis use further reduced the association between stimulant use and risk of schizophrenia (adjusted odds ratio 1.1, 0.6 to 2.1). The association observed between schizophrenia and frequency of cannabis use was unchanged after adjustment for stimulant use.

\section{Other psychoses}

A total of 446 subjects were admitted with other psychoses. Subjects who had ever used cannabis had an increased risk of developing a psychosis other than schizophrenia (crude odds ratio 1.4, 1.1 to 1.9 ), but this effect was reduced after adjustment (adjusted odds ratio $1.1,0.8$ to 1.5 ). A similar pattern was observed for the association with cannabis frequency, with a linear trend odds ratio of 1.1 (1.0 to $1.2, \mathrm{P}<0.02)$ before adjustment and of $1.0(0.9$ to $1.1, \mathrm{P}<0.85)$ after adjustment.

For all the analyses, diagnosis on conscription, IQ score, and place of upbringing contributed most to confounding. Adjusting for the other potential confounders made virtually no difference to the final adjusted results.
Table 2 Crude and adjusted odds ratios with 95\% confidence intervals for developing schizophrenia any time after conscription in subjects who have ever used cannabis

\begin{tabular}{|c|c|c|c|c|}
\hline \multirow[b]{2}{*}{ Drug use } & \multirow{2}{*}{$\begin{array}{c}\text { No of } \\
\text { subjects }\end{array}$} & \multirow{2}{*}{$\begin{array}{c}\text { No }(\%) \text { of subjects } \\
\text { developing } \\
\text { schizophrenia }\end{array}$} & \multicolumn{2}{|c|}{ Odds ratio $(95 \% \mathrm{CI})$} \\
\hline & & & Crude & Adjusted* \\
\hline Cannabis ever† & 5391 & $73(1.4)$ & $2.2(1.7$ to 2.8$)$ & $1.5(1.1$ to 2.0$)$ \\
\hline \multicolumn{5}{|l|}{ Frequency of use of cannabis (ever): } \\
\hline None & 36429 & $215(0.6)$ & $1.0 \dagger$ & $1.0 \dagger$ \\
\hline Once & 608 & $2(0.3)$ & $0.6(0.1$ to 2.2$)$ & 0.6 (0.1 to 2.3$)$ \\
\hline 2-4 times & 1380 & $8(0.6)$ & $1.0(0.5$ to 2.0$)$ & $0.9(0.4$ to 1.9$)$ \\
\hline $5-10$ times & 806 & $9(1.1)$ & $1.9(1.0$ to 3.7$)$ & $1.4(0.7$ to 2.8$)$ \\
\hline $11-50$ times & 689 & $13(1.9)$ & $3.2(1.8$ to 5.7$)$ & $2.2(1.2$ to 4.0$)$ \\
\hline$>50$ times & 731 & $28(3.8)$ & $6.7(4.5$ to 10.0$)$ & 3.1 (1.7 to 5.5$)$ \\
\hline Linear trend for frequency of use & - & - & $1.4(1.3$ to 1.5$)$ & $1.2(1.1$ to 1.4$)$ \\
\hline
\end{tabular}

${ }^{*}$ Adjusted for diagnosis at conscription to IQ score to poor social integration to disturbed behaviour to cigarette smoking to and place of upbringing.

†No drug use as baseline comparison.

Table 3 Crude and adjusted odds ratios with 95\% confidence intervals $(95 \% \mathrm{Cl})$ for developing schizophrenia in years $0-5$ after conscription in subjects who have ever used cannabis

\begin{tabular}{lcccc} 
& No of & $\begin{array}{c}\text { No (\%) of subjects } \\
\text { developing }\end{array}$ & \multicolumn{2}{c}{ Odds ratio (95\% Cl) } \\
\cline { 4 - 5 } Drug use & 5320 & $33(0.6)$ & $2.2(1.7$ to 2.8$)$ & $2.1(1.2$ to 3.7$)$ \\
\hline Subjects & schizophrenia & Crude & Adjusted $^{*}$ \\
\hline Frequency of use of cannabis (ever): & 36429 & $47(0.1)$ & $1.0 \dagger$ & $1.0 \dagger$ \\
\hline None & 608 & 0 & - & - \\
\hline Once & 1380 & $2(0.1)$ & $1.1(0.3$ to 4.6$)$ & $1.0(0.2$ to 4.4$)$ \\
\hline $2-4$ times & 806 & $4(0.5)$ & $3.9(1.4$ to 10.7$)$ & $2.6(0.8$ to 7.9$)$ \\
\hline $5-10$ times & 689 & $4(0.6)$ & $4.5(1.6$ to 12.6$)$ & $2.8(0.9$ to 8.8$)$ \\
\hline $11-50$ times & 731 & $13(1.8)$ & $14.0(7.5$ to 26.0$)$ & $4.7(1.8$ to 12.4$)$ \\
\hline$>50$ times & & - & $1.6(1.4$ to 1.8$)$ & $1.3(1.1$ to 1.6$)$ \\
\hline Linear trend for frequency of use & & & &
\end{tabular}

${ }^{*}$ Adjusted for diagnosis at conscription, IQ score, poor social integration, disturbed behaviour, cigarette smoking, and place of upbringing.

†No drug use as baseline comparison.

Table 4 Crude and adjusted odds ratios with $95 \%$ confidence intervals $(95 \% \mathrm{Cl})$ for developing schizophrenia in years $5+$ after conscription in subjects having ever used cannabis

\begin{tabular}{|c|c|c|c|c|}
\hline \multirow[b]{2}{*}{ Drug use } & \multirow{2}{*}{$\begin{array}{l}\text { No of } \\
\text { subjects }\end{array}$} & \multirow{2}{*}{$\begin{array}{c}\text { No }(\%) \text { of subjects } \\
\text { developing } \\
\text { schizophrenia }\end{array}$} & \multicolumn{2}{|c|}{ Odds ratio $(95 \% \mathrm{Cl})$} \\
\hline & & & Crude & Adjusted* \\
\hline Cannabis ever† & 5287 & $40(0.8)$ & 1.6 (1.1 to 2.2$)$ & $1.2(0.8$ to 1.8$)$ \\
\hline \multicolumn{5}{|l|}{ Frequency of use of cannabis (ever): } \\
\hline None & 36382 & $168(0.5)$ & $1.0 \dagger$ & $1.0 \dagger$ \\
\hline Once & 608 & $2(0.3)$ & 0.7 (0.2 to 2.9$)$ & 0.8 (0.2 to 3.2$)$ \\
\hline 2-4 times & 1378 & $6(0.4)$ & $0.9(0.4$ to 2.1$)$ & $0.9(0.4$ to 2.0$)$ \\
\hline 5-10 times & 802 & $5(0.6)$ & $1.4(0.6$ to 3.3$)$ & $1.0(0.4$ to 2.5$)$ \\
\hline $11-50$ times & 685 & $9(1.3)$ & $2.9(1.5$ to 5.6$)$ & 2.1 (1.0 to 4.5$)$ \\
\hline$>50$ times & 718 & $15(2.1)$ & 4.6 (2.7 to 7.8$)$ & $2.5(1.2$ to 5.1$)$ \\
\hline Linear trend for frequency of use & - & - & $1.3(1.2$ to 1.4$)$ & $1.2(1.0$ to 1.3$)$ \\
\hline
\end{tabular}

${ }^{*}$ Adjusted for diagnosis at conscription, IQ score, poor social integration, disturbed behaviour, cigarette smoking, and place of upbringing.

†No drug use as baseline comparison.

\section{Discussion}

Self reported use of cannabis in early adulthood was associated with an increased risk of developing schizophrenia. Risk increased in a dose dependent manner with increasing frequency of cannabis use, and this relation remained when analysis was restricted to subjects who had used only cannabis and no other drugs before conscription. The largest risk was seen in subjects reporting use of cannabis on more than 50 occasions. We found no association between cannabis and other psychotic illnesses, which implies that cannabis has a rather specific association with an increased risk of schizophrenia. 
Table 5 Adjusted odds ratios with 95\% confidence intervals for developing schizophrenia any time after conscription for subjects taking cannabis only

\begin{tabular}{|c|c|c|c|c|}
\hline \multirow[b]{2}{*}{ Drug use } & \multirow{2}{*}{$\begin{array}{c}\text { No of } \\
\text { subjects }\end{array}$} & \multirow{2}{*}{$\begin{array}{l}\text { No }(\%) \text { of subjects } \\
\text { developing } \\
\text { schizophrenia }\end{array}$} & \multicolumn{2}{|c|}{ Odds ratio $(95 \% \mathrm{CI})$} \\
\hline & & & Crude & Adjusted $^{*}$ \\
\hline Cannabis ever† & 1635 & $18(1.1)$ & $1.9(1.2$ to 3.0$)$ & $1.9(1.1$ to 3.1$)$ \\
\hline \multicolumn{5}{|l|}{ Frequency of use of cannabis (ever): } \\
\hline None & 36429 & $215(0.6)$ & $1.0 \dagger$ & $1.0 \dagger$ \\
\hline Once & 245 & 0 & - & - \\
\hline 2-4 times & 499 & $5(1.0)$ & $1.7(0.7$ to 4.2$)$ & $1.9(0.8$ to 4.8$)$ \\
\hline 5-10 times & 255 & $3(1.2)$ & $2.0(0.6$ to 6.3$)$ & $1.7(0.5$ to 5.7$)$ \\
\hline $11-50$ times & 176 & $1(0.6)$ & $1.0(0.1$ to 6.9$)$ & $0.8(0.1$ to 6.0$)$ \\
\hline$>50$ times & 70 & $4(5.7)$ & 10.2 (3.7 to 28.3 ) & 6.7 (2.1 to 21.7$)$ \\
\hline Linear trend for frequency of use & - & - & $1.3(1.1$ to 1.6$)$ & $1.3(1.0$ to 1.5$)$ \\
\hline
\end{tabular}

*Adjusted for diagnosis at conscription, IQ score, poor social integration, disturbed behaviour, cigarette smoking, and place of upbringing.

†No drug use as baseline comparison.

The association between use of cannabis and schizophrenia was stronger in subjects who were first admitted within five years of conscription. One explanation is that subjects with a prodrome of schizophrenia at conscription may have increased their cannabis use, perhaps as a means of self medication. ${ }^{2}$ But all subjects were screened at conscription, and we adjusted for other psychiatric problems recorded at that time. The relation with cannabis use was also observed in the later onset group, admitted more than five years after conscription. It seems more likely that the reduced association in the group with later onset is due to misclassification, as the number of people who discontinued cannabis use accumulated over time. ${ }^{22}$

Although adjustment for confounders substantially reduced the odds ratios, adjusting for poor social integration had only minimal effects. A similar effect was observed in the original study by Andreasson et al, who adjusted for the number of friends that the subjects reported having. ${ }^{4}$ We used a more comprehensive measure of social integration as it is likely that on its own this question was not a strong measure of sociable personality traits. Personality traits are difficult to measure accurately, however, and residual confounding remains a possibility. The association between cannabis and schizophrenia persisted even after adjusting for use of alcohol, cigarettes, and other drugs, all of which are likely to be indicative of risk taking behaviour. This implies that a shared risk factor (be it biological, genetic, or through personality traits) for developing schizophrenia and for using psychoactive substances does not adequately explain the association observed.

We are limited in that we have only data regarding use of cannabis before conscription. But if the pattern of increased initiation and reduced cessation of drug use seen in the schizophrenia group persisted after the time of conscription, this would result in us underestimating the effect size of cannabis. Fewer subjects in this cohort claimed to have used cannabis and other illicit drugs compared with similar cohorts that used anonymous questionnaires. ${ }^{23}$ The effect of under-reporting would again result in an underestimate of the true effect size. Non-response was similar for subjects developing schizophrenia and non-cases, although, as a further check, we repeated the analyses, having recoded non-responders as either users or non-users of cannabis. This made no difference when recoding was non-differential between cases and non-cases, but it
What is already known about this topic

Use of cannabis has been associated with an increased risk of developing schizophrenia

Alternative explanations for this association include confounding by personality or by use of other drugs such as amphetamines, and use of cannabis as a form of self medication secondary to the disorder

\section{What this study adds}

Self reported cannabis use is associated with an increased risk of subsequently developing schizophrenia, consistent with a causal relation

This association is not explained by sociability personality traits, or by use of amphetamines or other drugs

Self medication with cannabis is an unlikely explanation for the association observed

increased the odds ratios substantially when recoding was differential.

It is possible that use of stimulants could explain the results if stimulants were able to induce a chronic psychotic illness, identical to schizophrenia. But we did not find an independent association between use of stimulants and schizophrenia, although power was reduced compared with other analyses. Although studies from the United States have found that initiation of amphetamine use peaks by age $18-20,{ }^{22}$ it is possible that initiation of stimulants after conscription was more likely in subjects who had previously used only cannabis. But the absence of an independent association with use of stimulants in our data implies that cannabis is potentially the more important agent.

These findings are in keeping with accumulating evidence that cannabis has detrimental effects on mental health in some people. ${ }^{3}$ Molecular studies have shown that $\Delta^{9}$-tetrahydrocannabinol, the active component of cannabis, increases release of dopamine in the mesolimbic pathway. ${ }^{24}$ Given the suggested relation between increased mesolimbic dopamine and positive symptoms of schizophrenia, ${ }^{25}$ such observations provide support for the hypothesis that cannabis may act as a risk factor for this disorder.

Use of cannabis use has increased substantially over the past few decades in the United Kingdom, and $50 \%$ of the population now report having used cannabis at least once. ${ }^{26}$ If cannabis increases the risk of schizophrenia by $30 \%$, as implied by these results, then $13 \%$ of cases of schizophrenia could be prevented if cannabis use was eliminated from the population, assuming that a causal relation between cannabis use and schizophrenia really exists. The overall weight of evidence is that occasional use of cannabis has few harmful effects overall, ${ }^{2}$ and the drug is less likely to be used regularly and cause dependence than nicotine. Nevertheless, these results indicate a potentially serious risk to the mental health of people who use cannabis, particularly in the presence of other risk factors for schizophrenia. Such risks need to be considered in the current move to liberalise and possi- 
bly legalise the use of cannabis in the United Kingdom and other countries.

We thank Hollie Thomas and Diane McCracken for their help and advice, and Jonas Sadigh for his help with data management.

Contributors: SZ contributed to the conception of the study, data analysis, and drafting of the manuscript. PA contributed to the conception and design of the study, and drafting of the manuscript. SA contributed to the conception and design of the study, and drafting of the manuscript. IL contributed to the design of the study and drafting of the manuscript. GL contributed to the conception of the study, data analysis, and drafting of the manuscript. SZ is the guarantor.

Funding: This research is funded from a clinical training fellowship grant, awarded to SZ by the Medical Research Council, UK (grant no. G84/5689).

Competing interests: None declared.

1 Thomas H. Psychiatric symptoms in cannabis users. Br J Psychiatr 1993;163:141-9.

2 Hall W, Solowij N, Lemon J. The health and psychological consequences of cannabis use. Canberra: Australian Government Publishing Service, 1994.

3 Johns A. Psychiatric effects of cannabis. Br J Psychiatry 2001:178:116-22.

4 Andreasson S, Allebeck P, Engstrom A, Rydberg U. Cannabis and schizophrenia. A longitudinal study of Swedish conscripts. Lancet 1987;2:1483-6.

5 Johnson BA, Smith BL, Taylor P. Cannabis and schizophrenia. Lancet 1988; $1: 592-3$

6 Negrete JC. Cannabis and schizophrenia. Br J Addict 1989;84:349-51.

7 Hall W, Degenhardt L. Cannabis use and psychosis: a review of clinical and epidemiological evidence. Aust N Z J Psychiatry 2000;34:26-34.

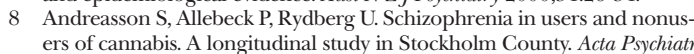
Scand 1989;79:505-10.

9 Johnstone EC. Schizophrenia. In: Johnstone EC, Freeman CPL, Zealley AK. Companion to psychiatric studies. 6th ed. London: Churchil Livingstone, 1998:369-97.

10 Lewis G, David A, Andreasson S, Allebeck P. Schizophrenia and city life Lancet 1992:340:137-40.
11 David AS, Malmberg A, Brandt L, Allebeck P, Lewis G. IQ and risk for schizophrenia: a population-based cohort study. Psychol Med 1997;27:1311-23.

12 Malmberg A, Lewis G, David A, Allebeck P. Premorbid adjustment and personality in people with schizophrenia. Br J Psychiatry 1998;172:308-

13 Lewis G, David AS, Malmberg A, Allebeck P. Non-psychotic psychiatric disorder and subsequent risk of schizophrenia. Cohort study. Br J Psychiatry 2000; $177: 416-20$.

14 Otto U. Function of male youths during military service. A follow-up study of a youth clientele. Acta Psychiatr Scand 1980;282 (suppl):1-60.

15 World Health Organization. Glossary of mental disorders and guide to their classification for use in conjunction with International Classification of Diseases. Eight revision. Geneva: WHO, 1974

16 Geddes JR, Kendell RE. Schizophrenic subjects with no history of admission to hospital. Psychol Med 1995;25:859-68.

17 Jablensky A. Epidemiology of schizophrenia: a European perspective. Schizophr Bull 1986;12:52-73.

18 Kristjansson E, Allebeck P, Wistedt B. Validity of the diagnosis of schizophrenia in a psychiatric inpatient register. Nordisk Psychiatrisk Tidskrift 1987;41:229-34

19 Greenland S, Rothman KJ. Introduction to categorical statistics. In: Rothman KJ, Greenland S. Modern epidemiology. 6th ed. Philadelphia: Lippincott Raven, 1998:231-52.

20 Zammit S, Allebeck P, Dalman C, Lundberg I, Owen M, Lewis G. Paternal age as a risk factor for schizophrenia (in press).

21 Zammit S, Allebeck P, Dalman C, Lundberg I, Lewis G. Investigating the association between cigarette smoking and schizophrenia using a cohort study (in press).

22 Chen K, Kandel DB. The natural history of drug use from adolescence to the mid-thirties in a general population sample. Am J Public Health 1995;85:41-7.

23 Andreasson S. Misuse of alcohol and cannabis among young men. Stockholm: Karolinska Institute, 1990.

24 Tanda G, Pontieri FE, Di Chiara G. Cannabinoid and heroin activation of mesolimbic dopamine transmission by a common mul opioid receptor mechanism. Science 1997;276:2048-50.

25 Davis KL, Kahn RS, Ko G, Davidson M. Dopamine in schizophrenia: a review and reconceptualization. Am J Psychiatry 1991;148:1474-86.

26 Singleton N, Bumpstead R, O'Brien M, Lee A, Meltzer H. Psychiatric morbidity among adults living in private households, 2000. London: Stationery Office, 2001.

(Accepted 12 September 2002) 